\title{
Étude de la dynamique spatio-temporelle de la réserve de la Comoé-Léraba et de ses terroirs environnants (Burkina Faso, Afrique de l'Ouest)
}

\author{
Assan GNOUMOU1* Aoupoaoune Basile ADOUABOU2 \\ 'Unité de Formation et de Recherche, Sciences Appliquées et Technologies, Université de Dédougou BP 176 Burkina \\ Faso.agnoumou@gmail.com. Cel : 76412924 \\ 2Department of environment and sustainable development, project BKF/015-IFN2, Ouagadougou, Burkina Faso.
}

Original submitted in on $1^{\text {st }}$ November 2020. Published online at www.m.elewa.org/journals/ on 31st January 2021 https://doi.org/10.35759/JABs.157.7

\section{RESUME :}

Objectif : L'emprise de l'Homme sur la végétation s'est traduite au cours de ces dernières décennies par une augmentation des superficies emblavées. Cette étude vise à analyser la dynamique spatio-temporelle de la végétation de la réserve de la Comoé-Léraba et ses terroirs sur une période de 20 ans.

Méthodologie et résultats : Deux types d'images Landsat TM, ETM+ pour les périodes de 1990 et 2000 et ASTER 2009 ; prises toutes au mois de novembre ont été nécessaires pour cette étude diachronique. Le logiciel GEOMATICA 9.2 de PCI a été utilisé pour le traitement des images. Ainsi, l'analyse diachronique de la végétation à l'intérieur de l'aire protégée sur les 20 ans témoigne d'une reconstitution du couvert végétale, avec une perte importante des savanes arbustives, des zones de culture et des zones nues (15,93 \%). Cependant, hors de l'aire protégée, la synthèse montre une augmentation importante des zones de cultures $(14,27 \%)$ au détriment des forêts claires, des savanes boisées et arbustives avec une réduction de leurs superficies $(15,37 \%)$.

Conclusion et applications des résultats : L'analyse de la dynamique de la végétation dans la réserve ComoéLéraba et dans ses terroirs, révèle que la pression anthropique menace sa végétation. En effet, cette étude a permis de mettre en évidence que dans les terroirs sous influence humaine, les superficies des formations végétales se réduisent lamentablement au profit des zones de cultures. A l'opposé, les hameaux de cultures abandonnés se sont véritablement reconstitués en savanes arbustives à l'intérieur de l'aire protégée. Même si de nouvelles taches de zones de culture ont commencé à apparaître dans la zone dite tampon, soit par empiètement sur les limites officielles, nous pouvons affirmer que la gestion participative a eu un effet positif dans la dynamique d'évolution des formations végétales dans la réserve de la Comoé-Léraba. Juste qu'à présent cette carte d'occupation des terres éditée de 2009 constitue la plus récente de la réserve.

Mot clés : télédétection, aire protégée, conservation, cartographie, végétation. 


\section{ABSTRACT \\ Spatial and temporal dynamics of the vegetation of Comoé-Léraba reserve and its surrounding lands (Burkina Faso, West Africa)}

Objectives: Over the last few decades, Human pressure on vegetation has resulted in an increase number of dispersed deforested area. This study aims to analyse the spatio-temporal dynamics of the vegetation in the Comoé-Léraba Reserve and its surrounding areas over a period of 20 years.

Methodology and Results: Two types of Landsat TM images, for the periods 1990 and 2000 and ASTER 2009; all taken in November were required for this study. PCl's GEOMATICA 9.2 software was used for image processing. Thus, the diachronic analysis of vegetation within the protected area over 20 years shows a reconstitution of the vegetation cover, with a significant loss of shrubby savannahs, cultivated areas and bare areas (15.93\%). However, outside the protected area for the same period, the synthesis shows a significant increase in cultivated areas (14.27\%) to the detriment of open forests, wooded and shrubby savannahs with a reduction in their surface area (15.37\%).

Conclusions and application of findings: The analysis of the dynamics of land use in the Comoé-Léraba Reserve and its surrounding area indicates that anthropic pressure threatens its vegetation. In fact, this study has shown that in areas under human influence, the surface area of plant communities is being reduced dramatically in favour of cultivated areas. On the other hand, the hamlets of abandoned crops have truly reconstituted themselves into shrubby savannahs within the protected area. Even if new patches of cultivated areas have begun to appear in the so-called buffer zone, either through encroachment on the official boundaries, we can affirm that participatory management has had a positive effect in the dynamics of the evolution of plant formations in the Comoé-Léraba Reserve. Up to now, this land use map published in 2009 is the most recent land use map for the reserve.

Key words: remote sensing protected area, conservation, mapping, vegetation.

\section{INTRODUCTION}

Les investigations sur le couvert végétal à un niveau local directement sur les sites restent laborieuses, fastidieuses et presque impossibles à grande échelle. Elles sont souvent subjectives car l'estimation d'une voûte peut varier selon l'appréciateur. Elles sont également limitées dans le temps et sur une petite échelle (Yang et al., 2012). Par ailleurs, la télédétection est une science qui donne une alternative pour quantifier les éléments biophysiques au niveau du sol (Conhen et al., 2003) à grande échelle. Elle permet en plus d'accéder aux informations portant sur leur état antérieur (Jones et al. 2019). Par conséquent, le suivi de la dynamique du couvert végétal dans le temps et dans l'espace, à l'aide d'images satellitaires est une précieuse contribution à la gestion des ressources naturelles (Leimgruber et al., 2005). Ce suivi offre des estimations concrètes du couvert végétal et le rythme de la déforestation. La télédétection est très utilisée dans l'étude des forêts tropicales qui sont fortement influencées par des processus bioclimatiques et anthropogènes (Dimobe et al.,
2015 ; Chirima et al, 2018 ; Belem et al. 2018 ; Sieza et al. 2019). Des études réalisées en Afrique de l'Ouest ont déjà montré que la déforestation se fait à une vitesse inquiétante (Ajonou et al. 2010 ; Biga et al. 2020). Les formations végétales du Burkina Faso subissent également l'impact négatif de ces deux facteurs dont l'une des conséquences est la régression du couvert végétal. Une cartographie de l'occupation des terres à partir d'images satellitaires LANDSAT TM de 1992 et de LANDSAT 7 ETM+ de 2002 a permis de quantifier le phénomène de déforestation sur le territoire burkinabé (Anonyme, 2006). Ce couvert forestier subit une diminution moyenne annuelle de 110500 ha, soit 4,04\% en moyenne par an, de 1992 à 2002 (Anonyme, 2006). Réduire la vitesse de cette déforestation, nécessiterait des investigations à deux niveaux : premièrement, une connaissance précise des superficies couvertes de végétation que nous perdons et le rythme de déforestation (Davidar et al., 2010); puis deuxièmement, maîtriser les causes à des échelles réduites. Les causes au 
niveau local peuvent varier d'un endroit à un autre (Abdou et al., 2019 ; Tsewoue et al. 2020) car les cultures et l'histoire d'un peuple ont un impact sur les activités humaines. Les images satellitaires restent des outils fiables dans l'analyse de la dynamique de la végétation dans le temps et dans l'espace (Kanembou et al., 2009 ; Ajonou et al., 2010). Ces études montrent qu'il y a beaucoup de changements qui s'opèrent particulièrement autour des aires protégées (Abdou et al., 2019) et même souvent à l'intérieur (Guinko, 1997 ; Belem et al. 2018). Et cela constitue un souci majeur pour la conservation des ressources naturelles de ces aires protégées. Située à l'extrême sud-ouest du pays, la Forêt Classée et Réserve Partielle de Faune de la Comoé-Léraba (réserve de la Comoé-Léraba), subit l'influence des populations riveraines qui sont essentiellement des agriculteurs. On assiste ainsi à l'extension des aires de cultures, qui s'installent au détriment des formations végétales naturelles. La disparition des formations végétales entraine non seulement la perte de la phytodiversité mais surtout

\section{MATERIEL ET METHODES}

Milieu d'étude : La forêt classée et réserve partielle de faune de la Comoé-Léraba se situe entre la latitude $10^{\circ} 2^{\prime}$ et $9^{\circ} 31^{\prime} \mathrm{N}$ et la longitude $4^{\circ} 55^{\prime}$ et $4^{\circ} 13^{\prime} \mathrm{W}$ (Figure 1). Localisée dans le sud-ouest du Burkina Faso, elle s'étend sur une superficie d'environ 125000 ha. Du point de vue phytogéographique, la réserve appartient au secteur sud- soudanien (Fontès \& Guinko, 1995). Le réseau hydrographique est assez important ; et elle a été celle de la biodiversité en général. Cette perte constitue une pression permanente sur les ressources spécifiques disponibles. II est alors nécessaire de fournir des informations fiables pour mieux orienter et définir les méthodes de gestion de ces formations végétales. Cette étude vise à analyser la dynamique spatio-temporelle de la végétation et de l'occupation des terres de la réserve de la Comoé-Léraba et ses terroirs environnants (10 kilomètres autour de l'aire protégée), à partir de données satellitaires de trois périodes bi-décennales (1990-2009). Les changements qui peuvent s'observer portent entre autres sur la forme et les attributs des unités de végétation et d'occupation des terres. Les objectifs spécifiques sont (1) d'analyser la dynamique de l'occupation des terres à l'intérieur de l'aire protégée et dans ses terroirs ; (2) de produire des cartes d'occupation des terres, pour servir d'outil d'aide à la prise de décision, dans le cadre de la gestion et la conservation des ressources naturelles de l'aire protégée.

effet, certifiée le 7 octobre 2009 comme site Ramsar. Selon les travaux menés par Guinko en 1997, sa végétation est de type savanicole. Toutefois, une des caractéristiques de la zone reste les îlots de forêts denses humides. Dans les terroirs, l'activité socioéconomique se présente comme une menace, car l'agriculture y est extensive et $99 \%$ de la population en pratique. 


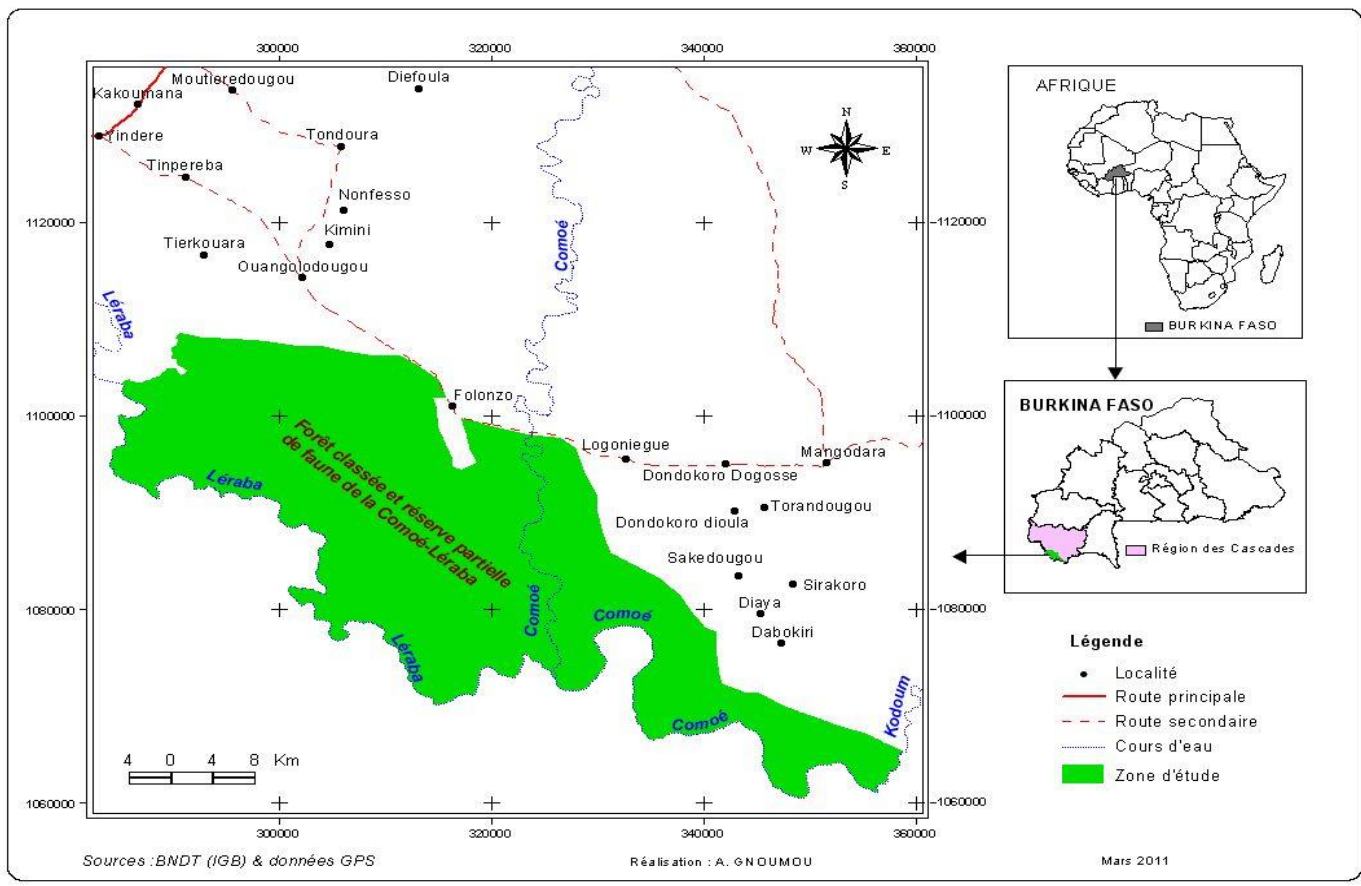

Figure 1 : Localisation de la zone d'étude

Démarche méthodologique: Deux types d'images couvrant la zone d'étude ont été utilisées à savoir les images Landsat TM, ETM ou ETM+ pour les périodes de 1990 et 2000. La moyenne résolution spatiale est de l'ordre de $30 \mathrm{~m}$. Puis une image ASTER pour la période de 2009 ayant $15 \mathrm{~m}$ de résolution spatiale. La période de prise des vues de toutes les images correspond au mois de novembre. Ces images de résolutions spatiale et spectrale voisines sont comparables. Les données vectorielles utilisées sont celles du fichier BNDT élaborés par l'IGB d'une part, pour vérifier les corrections

\section{RESULTATS}

Analyse de l'évolution des unités d'occupation dans la réserve de la Comoé-Léraba : Les graphiques des figures 2 et 3 montrent la proportion des unités déjà opérées sur les images ASTER 2009. D'autre part, ils ont été utilisés en superposition avec les résultats de traitement d'image, comme éléments d'occupation, en l'occurrence les localités, les réseaux hydrographique et routier. En plus, le traitement des images en vue de l'élaboration des bases de données d'occupation des terres (de 1990, 2000 et 2009) a eu lieu à l'issue de l'identification des classes d'occupation des terres et l'établissement des clés d'interprétation par classe en utilisant le logiciel de traitement d'images GEOMATICA 9.2 de PCl.

d'occupation des terres sur les trois périodes dans la réserve de la Comoé-Léraba et la dynamique de ces unités sur les 20 ans. 


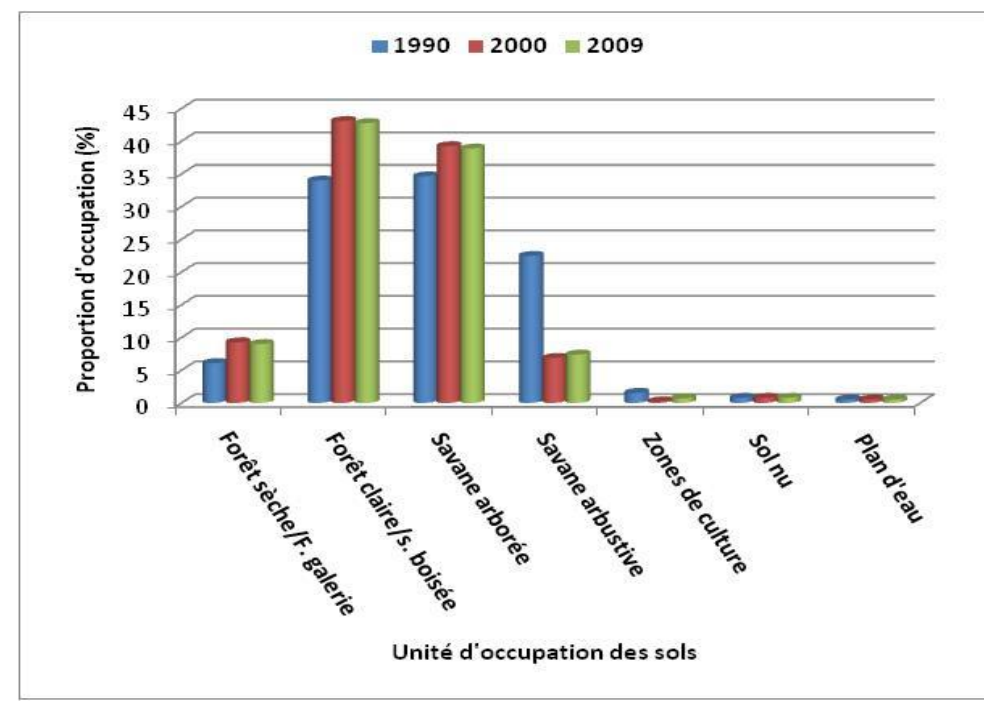

Figure 2 : Proportion des unités d'occupation des terres dans la Comoé-Léraba sur les trois périodes (années : 1990, 2000 et 2009)

Sur la base de l'interprétation des images satellitaires, il a y eu une évolution importante des superficies de presque toutes les unités d'occupation des terres les dix (10) premières années, à l'exception de l'unité des zones de cultures qui a connu une régression. En effet, les espaces occupés par les zones de cultures, abandonnés à la suite de la modification des limites de l'aire protégée et du changement de son statut, ont évolué en savanes arbustives ou en savanes arborées. De même, les autres unités ont également évolué vers des unités de strate supérieure (ex: savanes arbustives en savanes arborées, savanes arborées en savanes boisées). Cette reconstitution des unités de végétation de l'entité, semble être liée au mode de gestion (État et populations locales) mis en place pour la conservation des ressources naturelles en présence. Par contre dix (10) ans après, on constate une faible dégradation des ressources naturelles avec la réduction des superficies des unités de formations végétales au profit des zones de culture et les surfaces dénudées. Cela traduit un retour timide des agriculteurs dans cette aire protégée. Cette dégradation des ressources pendant cette période pourrait être aussi liée à un problème climatique, car l'étendue des plans d'eau a reculé pendant cette période.

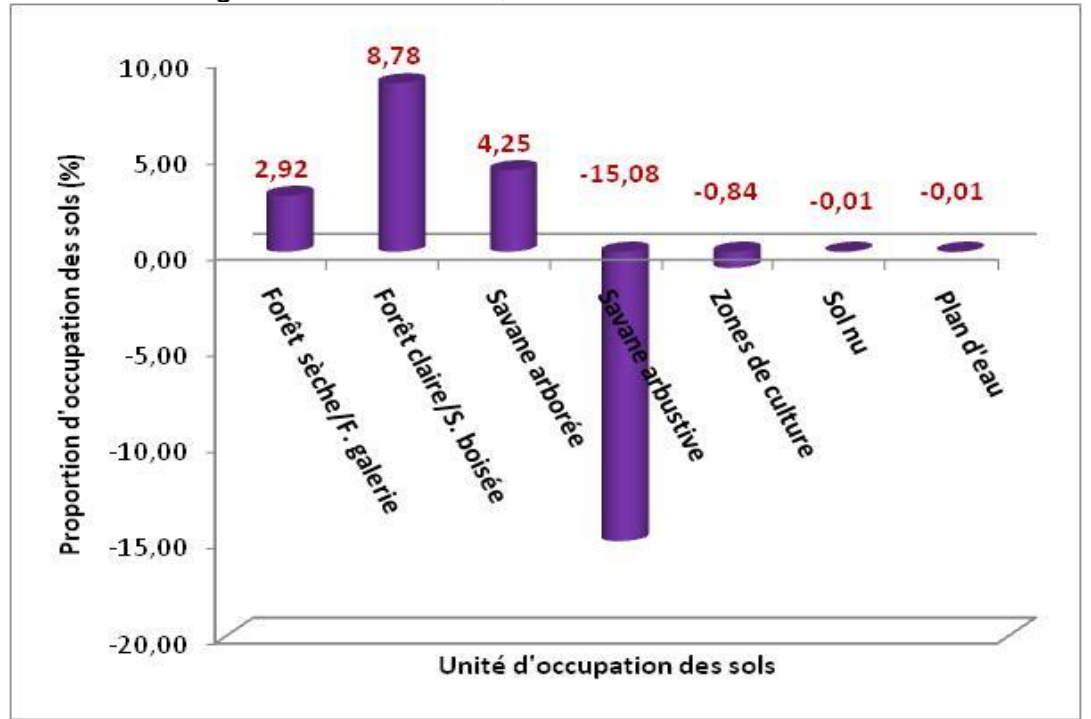

Figure 3 : Dynamique de l'occupation des terres dans la Comoé-Léraba sur les 20 ans 
Cependant, une analyse diachronique sur les 20 ans de l'image de 1990 et celle de 2009, révèle une tendance à la croissance de l'unité des forêts denses sèches et des forêts galeries, l'unité des savanes boisées et des forêts claires et les savanes arborées. Cela témoigne une reconstitution du couvert végétale dans l'aire protégée car parallèlement, il y a eu une perte importante des savanes arbustives, des zones de culture et des zones nues. Mais ce qui reste inquiétant, c'est la baisse de l'étendue des plans d'eau (Figure 3).

Analyse de l'évolution des unités d'occupation des terres dans les terroirs: L'analyse inter-période de 1990 et 2000, permet de constater une augmentation des superficies de certaines unités d'occupation des terres (figure 4). Les unités de végétation ayant enregistré un accroissement de leurs superficies sont les forêts denses sèches et les galeries forestières, les forêts claires et les savanes boisées ainsi que les savanes arborées. Cette situation pourrait être le résultat d'une reconstitution des ressources forestières au niveau de ces unités. Par contre, on note une augmentation des superficies des zones de culture correspondant à la disparition de formations végétales naturelles. En effet, à l'opposé des précédentes unités de végétation, celle des savanes arbustives a connu un recul considérable pendant cette même période. Ces types de formation furent certainement très prisés pour l'installation des champs. A la décade (2000-2009), on observe l'effet contraire car toutes les unités ont connu un recul de leur proportion en termes de superficies, à l'exception des zones de cultures qui continuent considérablement de gagner du terrain.

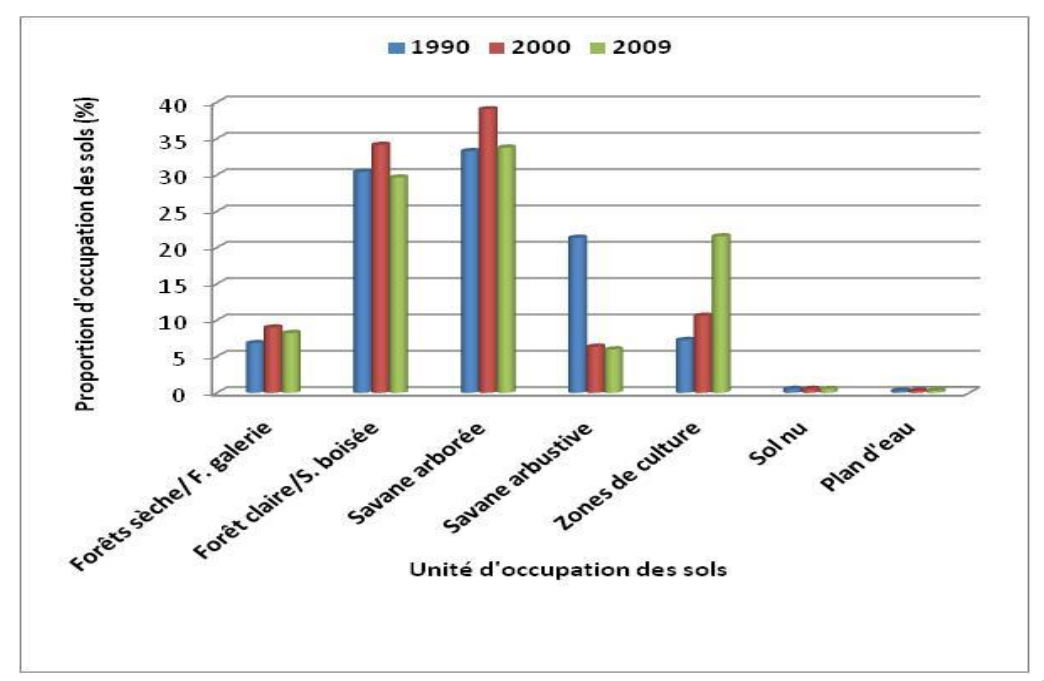

Figure 4 : Proportion des unités d'occupation des terres dans les terroirs sur les trois périodes (années : 1990, 2000 et 2009) 


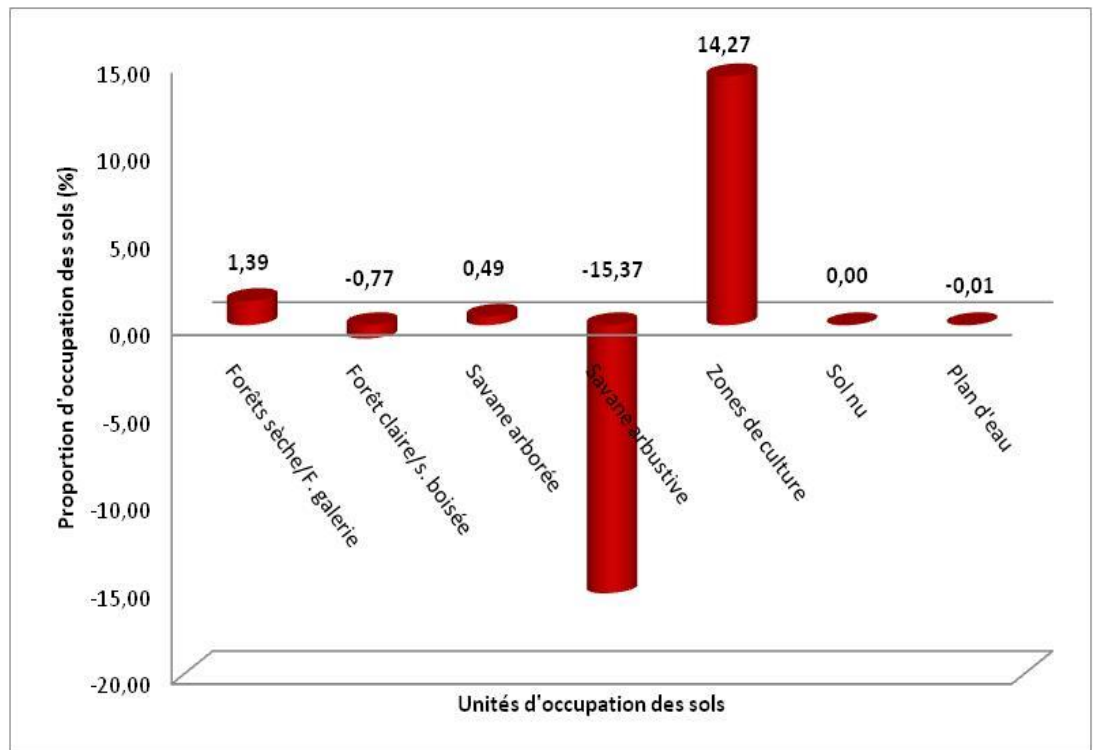

Figure 5 : Dynamique de l'occupation des unités de sols dans les terroirs sur les 20 ans

Ainsi la synthèse globale de la période de 20 ans (19902000), montre une augmentation importante des proportions des zones de cultures (Figure 5). On assiste aussi à une faible reconstitution de deux unités qui sont celles des forêts denses sèches et des galeries forestières puis celle des savanes arborées. Mais en réalité, seule l'augmentation de l'unité des forêts denses sèches et des galeries forestières peut être considérée comme un gain en ressources naturelles car l'augmentation des savanes arborées pourrait provenir de la dégradation des unités de végétation supérieures (forêts claires et savanes boisées). Pour ce qui concerne les unités des forêts claires et des savanes boisées, ainsi que celle des savanes arbustives, les tendances sont à la réduction de leurs superficies.
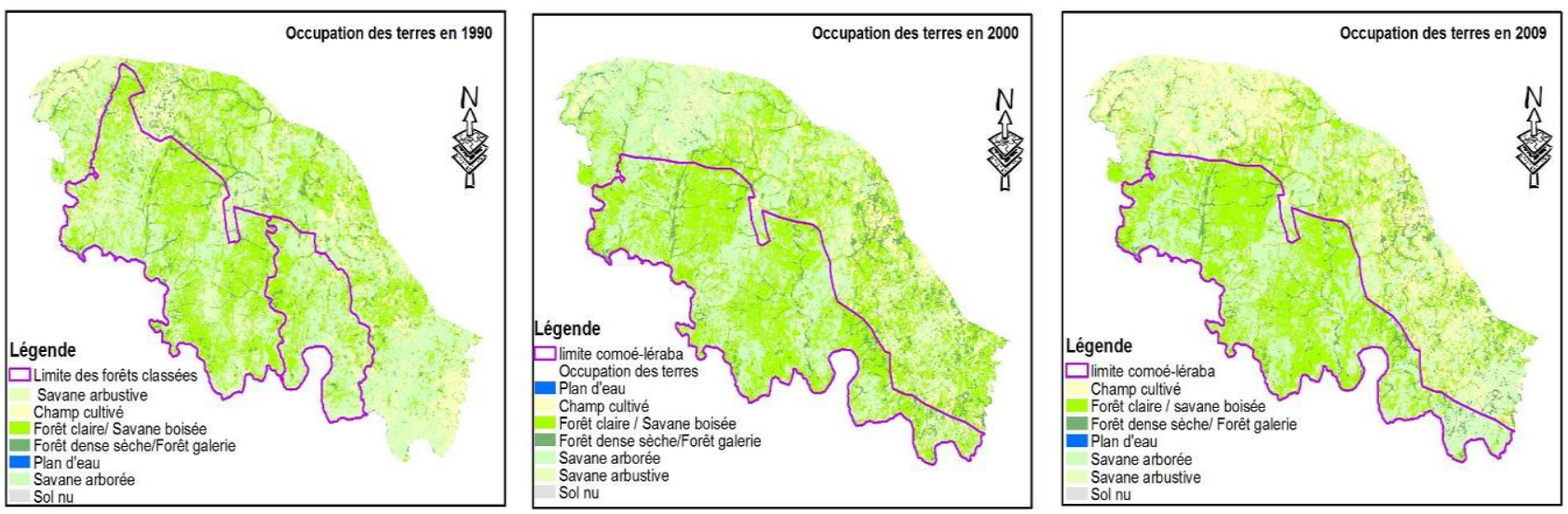

Figure 6 : Récapitulatif des cartes de l'évolution d'occupation des terres de 1990, 2000 et 2009.

\section{DISCUSSION}

Évolution globale du milieu naturel entre 1990 et 2009: Une forte pression était exercée sur les formations végétales des forêts classées de Diéfoula et de Logoniégué malgré leur statut d'aire protégée. En effet, une superficie de 3194,51 ha était occupée par les zones de culture. L'un des fardeaux de la conservation est l'exploitation illégale et frauduleuse des ressources naturelles que l'on tente de protéger (Gavin et al., 2010). Plusieurs approches de la conservation ont été adoptées par les gestionnaires des aires protégées pour pallier cette pratique. Mais depuis ces vingt (20) dernières années, l'approche participative semble être la solution, la mieux adaptée (Gbedahi et al., 2019) dans les aires protégées des pays en voie de développement. Ainsi, 
tout comme le Projet de Restauration des Ressources Forestières de Bassila (PRRF) au Bénin (Gbedahi et al., 2019), la végétation a connu évolution positive sous la gestion de l'AGEREF. D'une manière générale, cette étude révèle un recul considérable des zones de culture et des savanes arbustives dans la réserve et cela témoigne d'une bonne reconstitution. Cependant, il y a lieu de redoubler de vigilance car on note une reprise timide des cultures en 2009 dans les zones dites tampons. Les formations les plus convoitées se réduisent de plus en plus dans les terroirs. Ainsi les agriculteurs à la conquête de nouvelles terres tentent une fois de plus de s'orienter vers la zone protégée.

Influence de la pression anthropique sur la dynamique de la végétation de la Comoé-Léraba et de ses terroirs: Les zones de culture ont une croissance exponentielle dans les terroirs. L'analyse de la dynamique des unités d'occupation des terres montre aussi clairement la transformation d'une proportion de chaque unité de formation végétale en zones de culture. La pression anthropique sur le couvert végétal se caractérise par l'exploitation incontrôlée des formations végétales. Elle se caractérise par la pratique de l'agriculture extensive (Tsewoue et al., 2020) sur brûlis (défrichements, coupes sélectives et abusives...), les feux tardifs occasionnés par le braconnage, le piétinement lors des pâtures (Belem et al., 2018), la cueillette et les prélèvements mettant en péril la survie de nombreuses espèces d'où celle des communautés végétales (Dimobe et al., 2015). Ces pratiques existaient jadis certes toutefois les populations parvenaient à vivre en harmonie avec la nature. Mais de nos jours la croissance démographique (Munsi et al., 2010) et l'évolution du cheptel constituent une menace sur le milieu naturel (Kanembou et al., 2009). En 1985, la zone d'étude comptait 8482 habitants. Cette population est passée à 16148 en 1996 (selon le recensement général de la population), puis le taux d'accroissement prévoyait 36509 habitants en 2010 (Anonyme, 2000). La zone accueille également des agriculteurs en provenance d'autres régions à la recherche de nouvelles terres cultivables. La principale culture de rente dans la région est l'igname. Pour la culture des ignames, les cultivateurs préfèrent les nouvelles formations de forêts claires ou de savanes boisées. Les savanes arbustives ou arborées sur sols à texture sableuse sont très sollicitées pour la culture des arachides et du sésame. Cependant nous ne pouvons pas perdre de vue le fait que l'exploitation exagérée de ces formations végétales peut conduire à leur raréfaction, voire leur disparition à long terme (Kanembou et al., 2009). Le couvert végétal peut aussi se dégrader sous le poids des pâtures (Hamandawana, 2011 ; Sieza et al., 2019). L'élevage est pratiqué dans la zone par les Peuhls, installés dans presque tous les villages de l'AGEREF ou en transit avec le bétail en direction de la Côte d'Ivoire. Ainsi, le bétail broute la régénération naturelle (rejets et semis) de plusieurs espèces ligneuses (Afzelia africana, Khaya senegalensis, Uvaria chamea, Pterocarpus erinaceus) empêchant ainsi la survie de leurs peuplements.

Influence du climat sur la dynamique de la végétation de la Comoé-Léraba et de ses terroirs : Le recul des plans d'eau a été constaté dans l'aire protégée ainsi que dans les terroirs, ces vingt (20) dernières années. Cela pourrait s'expliquer à partir des données pluviométriques de la météo de Niangoloko et de Mangodara. II y a une diminution de la quantité de pluie tombée ces 10 dernières années par rapport aux précédentes. De même, ces 5 dernières années, les mois de novembre qui correspondent aux dates de prise de différentes images satellitaires ne sont plus pluvieux. A cette baisse et mauvaise répartition des pluies dans le temps s'ajoute le réchauffement mondial (IPCC, 2007) du climat entrainant une évaporation rapide des plans d'eau. Selon les travaux de Millogo (2001), ces aléas climatiques sont ressentis au Burkina Faso depuis près de 40 ans. Ils se sont traduits par les fréquentes sècheresses qui ont eu lieu $(1968,1973,1984)$ et également l'assèchement du climat qui se traduit par la descente vers le sud des isohyètes 600 et 900 en 30 ans (Millogo, 2001). L'impact du climat est plus général et même transfrontalier (Hannah, 2009). Son effet affecte les formations végétales dans l'aire protégée comme dans les terroirs. Les variations climatiques sont citées parmi les facteurs qui induisent la disparition des espèces végétales ( $\mathrm{Da}, 2010$; Belem et al. 2018). Cette disparition entraine alors la forte dégradation des ressources végétales ainsi que la régression du couvert végétal. Ceci pourrait expliquer la régression naturelle de certaines formations dans l'aire protégée, comme le cas de l'unité des galeries forestières et des forêts denses sèches. Nous avons pu constater sur le terrain la mort et la chute des arbres de certains îlots de forêts denses sèches à Anogeissus leiocarpa et à Guibourtia copallifera. Dans de rares cas, l'îlot tout entier est détruit sans l'intervention de l'homme. II y a également la chute de grands arbres (Dialium guineense) dans les galeries. Cette chute est induite par une montée brusque des eaux de pluie qui stagnent plus longtemps qu'auparavant. L'impact de la foudre sur la destruction de nombreux grands arbres fut très remarquable sur certaines forêts claires à Isoberlinia doka en 2008. 


\section{CONCLUSION}

L'étude diachronique de la végétation de la ComoéLéraba et ses terroirs a révélé des changements profonds dans sa dynamique en vingt (20) ans. Certaines formations sont restées intactes, d'autres ont été restaurées et par contre d'autres ont subi une dégradation profonde. L'étude montre que la pression anthropique est très forte sur les ressources végétales autour de cette aire protégée et la réserve de la ComoéLéraba elle-même n'est pas à l'abri de nouvelles

\section{REMERCIEMENTS}

Nous remercions Pr Adjima THIOMBIANO pour son encadrement scientifique, le projet BIOTA Afrique de I'Ouest (01 LC0617D1) qui dans sa Phase III a financé

\section{REFERENCES BIBLIOGRAPHIQUES}

Abdou IK, Abasse T, Massaoudou M, Rabiou H, Soumana I, Bogaert J, 2019. Influence des Pressions Anthropiques sur la Dynamique Paysagère de la Reserve Partielle de Faune de Dosso (Niger). Int. J. Biol. Chem. Sci. 13(2) : 1094-1108.

Adjonou K, Djiwa O, Kombate Y, Kokutse AD, Kokou K, 2010. Étude de la dynamique spatiale et structure des forêts denses sèches reliques du Togo : implications pour une gestion durable des aires protégées. Int. J. Biol. Chem. Sci. 4(1) : 168-183.

Anonyme 2000. Plan d'aménagement et de gestion de la forêt classée et réserve partielle de faune de la Comoé- Léraba, $68 \mathrm{p}$

Anonyme 2006. Évolution de l'occupation des terres entre 1992 et 2002 au Burkina Faso. Deuxième programme national de gestion des terroirs (PNGT2), Ouagadougou, $30 \mathrm{p}$.

Belem M, Zoungrana M, Nabaloum M, 2018. Les effets combinés du climat et des pressions anthropiques sur la forêt classée de Toéssin, Burkina Faso. Int. J. Biol. Chem. Sci. 12(5): 2186-2201.

Biga I, Amani A, Soumana I, bachir M, Mahamane A, 2020. Dynamique spatio-temporelle de l'occupation des sols des communes de Torodi, Gothèye et Tagazar de la région de Tillabéry au Niger. Int. J. Biol. Chem. Sci. 14 (3) : 949-965.

Chirima A, Mundy P, Ncube N, VanRooyen AF, 2018. Vegetation Changes in the Miombo Woodlands in Northwestern Zimbabwe: A Case Study of Nkayi District 1990 to 2017. Vegetation, Allan invasions. Toutefois, les cartes d'occupation des terres actualisées de 2009 restent des outils de gestion fiables disponibles. A cela les acteurs de prise de décision devraient veiller au contrôle de la croissance démographique et à l'éveil des consciences quant à l'utilisation rationnelle et judicieuse des ressources disponibles, de telle sorte que le système global dans lequel les écosystèmes cohabitent puisse maintenir son équilibre total.

ces travaux et l'AGEREF à travers son secrétariat exécutif pour leur soutien logistique ainsi que leur franche collaboration.

Sebata, IntechOpen, PP 43-57. http://dx.doi.org/10.5772/intechopen.72466

Cohen WB, Maiersperger TK, Gower ST, Turner DP, 2003. An improved strategy for regression of biophysical variables and Landsat ETM+ data. Remote Sensing of Environment, 84: 561-571.

Da SS, 2010. Spatial patterns of West-African plant diversity along a climatic gradient from coast to Sahel. Dissertation. University of Bonn. 131 pages.

Davidar P, Sahoo S, Mammen CP, Acharya P, Puyravaud JP, Arjunan M, Garrigues JP, Roessingh K, 2010. Assessing the extentand causes of forest degradation in India : Where do we stand? Biological Conservation (143): 2937-2944.

Dimobe K, Ouédraogo A, Soma S, Goetze D, Porembski S, Thiombiano A, 2015. Identification of driving factors of land degradation and deforestation in the Wildlife Reserve of Bontioli (Burkina Faso, West Africa). Global Ecology and Conservation 4: 559-571.

Fontès J. and Guinko S, 1995. Carte de la végétation et de l'occupation du sol du Burkina Faso. Note explicative. Ministère de la Coopération Francaise, Toulouse, France, 67p.

Gavin M, Solomon J, Blank A, 2010. Measuring and Monitoring Illegal Use of Natural Resources. Review. Conservation Biology. Vol (24), No.1

Gbedahi OLC, Biaou SSH, Mama A, Gouwakinnou GN, Yorou NS, 2019. Dynamique du couvert végétal à Bassila au nord Bénin pendant et après la mise en œuvre d'un projet d'aménagement 
forestier. Int. J. Biol. Chem. Sci. 13(1) : 311324.

Guinko S, 1997. Caractérisation des unités de végétation et appréciation de la diversité faunique de la zone d'intervention du projet GEPRENAF. Ministère de l'environnement et de l'eau, $74 \mathrm{p}$.

Hamandawana $\mathrm{H}, 2011$. The impacts of herbivory on vegetation in Moremi Game Reserve, Botswana: 1967-2001. Reg Environ Change. DOI 10.1007/s10113-011-0230-0

Hannah L, 2009. A global Conservation System for Climate-Change Adaptation. Conservation Biology, Vol. 24, No.1, 70-77.

IPCC, 2007. Fourth assessment Report 2007.

Jones PJ, Williamson GJ, David M. J. S. Bowman DMJS, Edward C, Lefroy EC, 2019. Mapping Tasmania's cultural landscapes: Using habitat suitability modelling of archaeological sites as a landscape history tool. Journal of Biogeography $46: 2570-2582$.

Kanembou L, Ambouta KJM, Waziri MM, 2009. Dynamique des aires pastorales dans le département de Gouré: apport de la télédétection et du SIG. Rev. Sc. Env. Uni., Lomé (Togo). N 005. ISSB 1812-1403.

Leimgruber $P$, Kelly DS, Steininger MK, Brunner J, Müller T, Songer M, 2005. Forest cover change patterns in Myanmar (1990-2000). Environmental Conservation, 32: 356-364.

Millogo-Rasolodimby J, 2001. L'homme, le climat et les ressources alimentaires végétales en période de crise de subsistance au Burkina Faso. Thèse de Doctorat d'état, Université de Ouagadougou, $250 \mathrm{p}$.

Munsi M, Areendran G, Joshi PK, 2012. Modeling spatiotemporal change patterns of forest cover: a case study from the Himalayan foothills (India). Reg Environ Change. DOI 10.1007/s10113011-0272-3

Sieza Y, Gomgnimbou APK, Serme I, Belem A, 2019. Étude de la variabilité climatique sur la dynamique d'occupation et d'utilisation des terres à des fins agro-pastorales dans la zone sud-soudanienne du Burkina Faso. Int. J. Biol. Chem. Sci. 13(4) : 1980-1994.

Tsewoue MR, Tchamba M, Avana ML, Tanougong AD, 2020. Dynamique spatio-temporelle de l'occupation du sol dans le Moungo, Région du Littoral, Cameroun : influence sur l'expansion des systèmes agroforestiers à base de bananiers. Int. J. Biol. Chem. Sci. 14 (2): 486500.

Yang J, Peter J, Weisberg PJ, Bristow NA, 2012. Landsat remote sensing approaches for monitoring long-term tree cover dynamics in semi-arid woodlands: Comparison of vegetation indices and spectral mixture analysis. Remote Sensing of Environment 119: 62-71. 